\title{
Inhibition of adenine nucleotide translocator pore function and protection against apoptosis in vivo by an HIV protease inhibitor
}

\author{
Joel G.R. Weaver, ${ }^{1,2,3}$ Agathe Tarze, ${ }^{4}$ Tia C. Moffat, ${ }^{5}$ Morgane LeBras, ${ }^{4}$ Aurelien Deniaud, ${ }^{4}$ \\ Catherine Brenner, ${ }^{4}$ Gary D. Bren, ${ }^{2}$ Mario Y. Morin, ${ }^{5}$ Barbara N. Phenix, ${ }^{3}$ Li Dong, ${ }^{3,6}$ \\ Susan X. Jiang, ${ }^{7}$ Valerie L. Sim, ${ }^{8}$ Bogdan Zurakowski, ${ }^{7}$ Jessica Lallier, ${ }^{5}$ Heather Hardin, ${ }^{9}$ \\ Peter Wettstein, ${ }^{9}$ Rolf P.G. van Heeswijk, ${ }^{3}$ Andre Douen, ${ }^{3}$ Romano T. Kroemer, ${ }^{10}$ Sheng T. Hou, ${ }^{7}$ \\ Steffany A.L. Bennett, ${ }^{5}$ David H. Lynch, ${ }^{11}$ Guido Kroemer, ${ }^{12}$ and Andrew D. Badley ${ }^{2,13}$

\begin{abstract}
${ }^{1}$ Division of General Surgery, University of Ottawa, Ottawa, Ontario, Canada. ${ }^{2}$ Division of Infectious Diseases, Mayo Clinic and Foundation, Rochester, Minnesota, USA. ${ }^{3}$ Ottawa Health Research Institute, University of Ottawa, Ottawa, Ontario, Canada. ${ }^{4}$ Université de Versailles Saint-Quentin-en-Yvelines, Versailles, France. ${ }^{5}$ Neural Regeneration Laboratory, Department of Biochemistry, Microbiology, and Immunology, and ${ }^{6}$ Department of Pathology, University of Ottawa, Ottawa, Ontario, Canada. ${ }^{7}$ Experimental Stroke Group, National Research Council Institute for Biological Sciences (NRC/IBS), National Research Council of Canada, Ottawa, Ontario, Canada. ${ }^{8}$ Division of Neurology, Ottawa Hospital, Ottawa, Ontario, Canada. ${ }^{11}$ Amnis Corp., Seattle, Washington, USA. ${ }^{12}$ Institut Gustave Roussy, Villejuif, France. ${ }^{13}$ Translational Program in Immunology and Biodefense, Mayo Clinic and Foundation, Rochester, Minnesota, USA.
\end{abstract} \\ ${ }^{9}$ Department of Surgery and Immunology, Mayo Clinic, Rochester, Minnesota, USA. ${ }^{10}$ Sanofi Aventis, Centre de Recherche de Paris, Vitry-sur-Seine, France.
}

Inhibitors of HIV protease have been shown to have antiapoptotic effects in vitro, yet whether these effects are seen in vivo remains controversial. In this study, we have evaluated the impact of the HIV protease inhibitor (PI) nelfinavir, boosted with ritonavir, in models of nonviral disease associated with excessive apoptosis. In mice with Fas-induced fatal hepatitis, Staphylococcal enterotoxin B-induced shock, and middle cerebral artery occlusion-induced stroke, we demonstrate that PIs significantly reduce apoptosis and improve histology, function, and/or behavioral recovery in each of these models. Further, we demonstrate that both in vitro and in vivo, PIs block apoptosis through the preservation of mitochondrial integrity and that in vitro PIs act to prevent pore function of the adenine nucleotide translocator (ANT) subunit of the mitochondrial permeability transition pore complex.

\section{Introduction}

The abnormal regulation of apoptosis is thought to contribute to a variety of pathologic disease processes in vivo. HIV-induced $\mathrm{CD} 4 \mathrm{~T}$ cell depletion and consequent immunodeficiency is one such disease state in which excessive apoptosis has been implicated. Current therapies for HIV not only reduce HIV replication but may also directly impact apoptosis; indeed, many groups have now reported that HIV protease inhibitors (PIs) can inhibit apoptosis at concentrations similar to those that are commonly seen in the plasma of patients receiving such treatments (reviewed in ref. 1). Paradoxically, such agents may also induce apoptosis, particularly of transformed cells, when used at higher doses (2-5).

Studies by several groups have investigated the potential mechanisms by which PIs may inhibit apoptosis, yielding different results. Proposed mechanisms include altered transcriptional regulation of key apoptosis regulatory proteins (5-7) and/or direct inhibition of the apoptosis enzyme ICE $(8,9)$ and/or calpain $(10)$. Such theories cannot fully account for the ability of PIs to inhibit diverse

\footnotetext{
Nonstandard abbreviations used: $\Delta \Psi_{\mathrm{m}}$, membrane permeability; ANT, adenine nucleotide translocator; AST, aspartate amino transferase; ATR, atractyloside; D-gal, D-galactosamine; MCAO, middle cerebral artery occlusion; NFV, nelfinavir; PBR, benzodiazepine receptor; PI, protease inhibitor; PTPC, permeability transition pore complex; RIT, ritonavir; RT, room temperature; SEB, Staphylococcal enterotoxin B; STS, staurosporine; TTC, 2,3,5-triphenyltetrazolium; VDAC, voltage-dependent anion channel.

Conflict of interest: The authors have declared that no conflict of interest exists.

Citation for this article: J. Clin. Invest. 115:1828-1838 (2005).

doi:10.1172/JCI22954.
}

apoptotic stimuli (reviewed in ref. 1) or the lack of enzymatic inhibition of recombinant caspases in vitro (11). According to another proposed mechanism to account for apoptosis inhibition, PIs alter the propensity of mitochondria to transduce apoptotic signals. This latter model is supported by the findings that PIs are able to block Fas-induced apoptosis involving mitochondrial signaling but not Fas-induced apoptosis that is mitochondria independent (11) and that PIs are able to rescue cells from apoptosis induced by mitochondriotoxic agents $(5,12)$.

Despite these in vitro findings, it remains controversial whether PI therapy for HIV-infected patients offers additional benefits in terms of CD4 T cell reconstitution compared with non-PI-containing regimens of equal antiviral potency $(13,14)$. Most studies that demonstrate enhanced CD4 $\mathrm{T}$ cell improvements in patients receiving PI therapy were retrospective, post-hoc analyses (15), which raises concerns about the methodologies used. Consequent$\mathrm{ly}$, at least one study was designed to compare CD4 T cell number, activation profile, memory and naive $\mathrm{T}$ cell subsets, and apoptosis between patients receiving PI-continuing or PI-sparing regimens (16). No differences were observed between groups regarding CD4 $\mathrm{T}$ cell number, activation, or memory or naive subsets; however, within the first week of therapy, significantly less apoptosis was seen in CD4 T cells of patients receiving PI therapy than in patients who did not receive PI therapy (16). Such data are consistent with the postulated antiapoptotic effects of PIs.

The objectives of this study were, first, to evaluate whether PIs were antiapoptotic in vivo by evaluating apoptotic changes in animal models of disease that are associated with excessive apopto- 

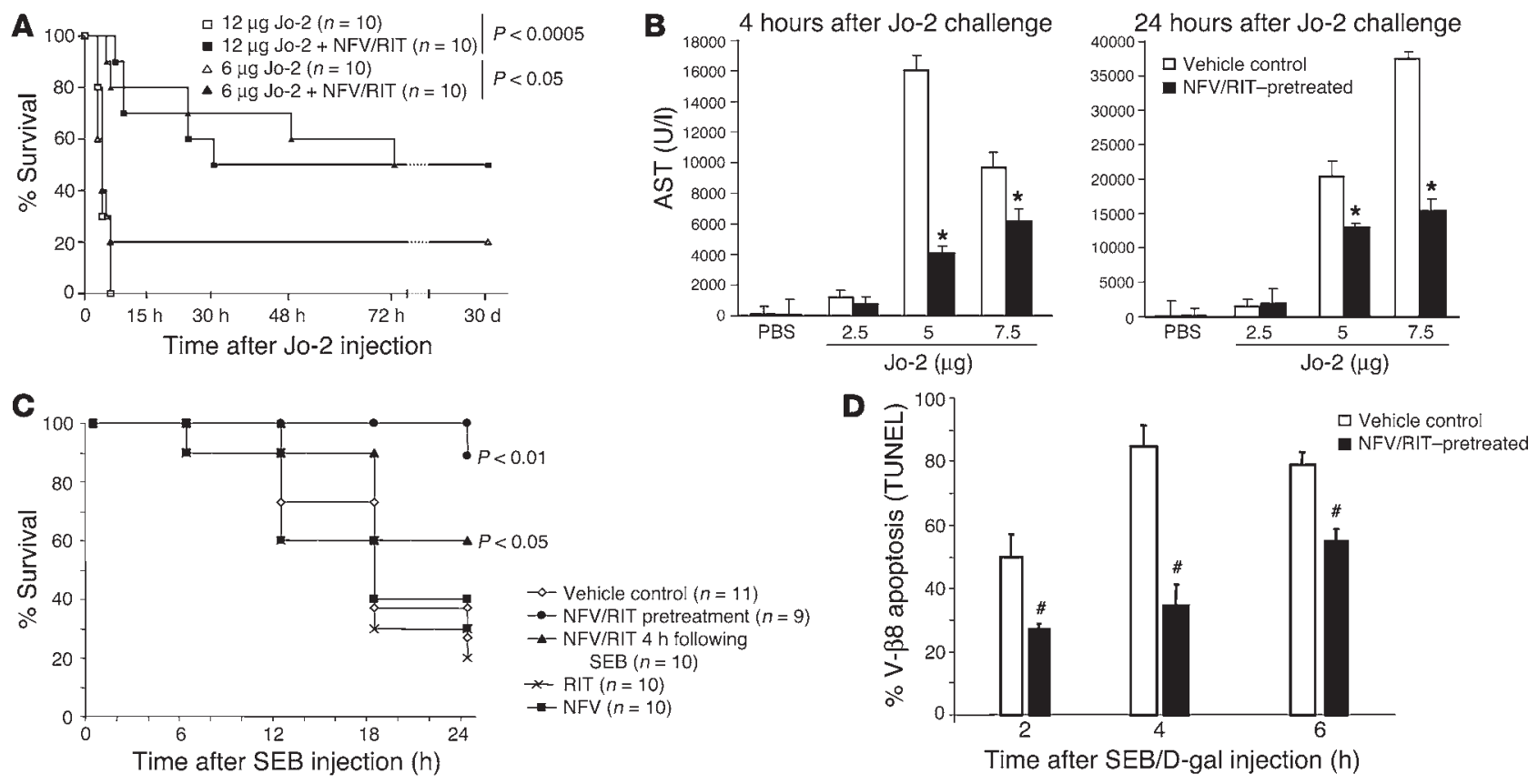

Figure 1

Effects of NFV/RIT on Jo-2-induced hepatitis or SEB-induced shock. (A) Mice treated with varying doses of Jo-2 antibody in the presence or absence of NFV/RIT were followed for 30 days and analyzed for survival. (B) In parallel, mice treated in a similar manner were sacrificed at 4 or 24 hours and analyzed for serum AST level. ${ }^{*} P<0.05$. (C) Five mice per group were treated with SEB/D-gal with or without NFV/RIT before or 4 hours after SEB or with NFV alone or RIT alone before SEB. Twenty-four-hour survival was monitored (cumulative data from 4 independent experiments are shown). (D) Mice treated with SEB/D-gal with NFV/RIT or control were analyzed for V- $\beta 8$ T cell apoptosis by TUNEL assay (cumulative data from 4 independent experiments are shown). ${ }^{*} P<0.01$.

sis but that do not depend upon viral replication and, second, to evaluate the mechanisms involved.

\section{Results}

As mouse metabolism of PIs differs from that of humans, we first performed pharmacokinetic studies of mice treated with nelfinavir (NFV) at doses used in human therapy. Within 1 hour of dosing, mice had undetectable levels of NFV. Therefore, we co-dosed mice with ritonavir (RIT), another PI known to increase PI levels in humans (17). Ultimately, a dose of $125 \mathrm{mg} / \mathrm{kg} \mathrm{NFV}$ and $13 \mathrm{mg} / \mathrm{kg}$ RIT resulted in drug levels similar to those of humans treated with NFV alone (see Methods). This dose was used for in vivo testing.

First, we evaluated the impact of NFV/RIT treatment on CD95/ Fas-induced hepatic failure (18-20). Mice received NFV/RIT or vehicle control pretreatment for 24 hours followed by treatment with 6 or $12 \mu \mathrm{g}$ of IV Jo- 2 anti-Fas antibody. Control animals died in a dose-dependent manner, whereas NFV/RIT-pretreated animals displayed superior survival compared with controls (Figure 1A). Moreover, survival of mice treated with RIT $(13 \mathrm{mg} / \mathrm{kg})$ was similar to that of controls, which indicates that NFV was responsible for the observed improved survival. Importantly, all mice that died did so within 72 hours, which indicates that NFV/RIT truly prevents rather than delays Jo-2-induced hepatotoxicity and death. In parallel, groups of 10 mice received $2.5,5$, or $7.5 \mu \mathrm{g}$ of IV Jo-2 with or without NFV/RIT pretreatment. Mice were sacrificed at 4 or 24 hours and analyzed for serum biochemistry, H\&E histology, and apoptosis by TUNEL staining. Serum glucose, blood urea nitrogen, creatinine, phosphorus, total protein, albumin, globulin, bilirubin, and cholesterol levels were similar in the 2 groups (data not shown).
However, NFV/RIT-pretreated mice had attenuated elevations in serum aspartate amino transferase (AST) at both 4 and 24 hours compared with control mice $(P<0.03$; Figure $1 \mathrm{~B})$, reduced evidence of hepatitis at 48 hours on H\&E histology, and a reduced quantity of TUNEL-positive hepatocytes from a mean of 50\% to $15 \%$ (measured in 5 mice per group; $P=0.002$; data not shown).

To assess whether NFV/RIT protection extended to other apoptotic disease processes, we treated mice with the bacterial superantigen Staphylococcal enterotoxin B (SEB) in the presence or absence of NFV/RIT. Systemic treatment with SEB results in shock and the selective apoptosis of $\mathrm{V}$ - $\beta 8$-positive $\mathrm{T}$ cells $(21)$. When coadministered with D-galactosamine (D-gal), SEB injection also results in death $(22,23)$. Following administration of SEB, apoptosis was measured by TUNEL assay in the V- $\beta 8$ and V- $\beta 3$ subsets of splenocytes. Four hours following SEB treatment, $72.6 \%$ of $\mathrm{V}$ - $\beta 8$ cells were TUNEL positive, whereas $21.7 \%$ of $\mathrm{V}-\beta 3$ cells were TUNEL positive $(n=3 ; P<0.05)$, which indicates that SEB selectively induced death in $\mathrm{V}-\beta 8$ cells. Parallel groups of mice were pretreated for 24 hours with NFV/RIT, vehicle control, NFV alone, or RIT alone, followed by treatment with $20 \mathrm{mg}$ D-gal and $6.5 \mu \mathrm{g}$ SEB. NFV/RIT-treated mice had improved survival compared with vehicle control ( $89 \%, n=9$ vs. $27 \%, n=11 ; P<0.01)$. Survival of vehicle-treated mice was not significantly different from that of mice treated with NFV alone $(30 \% ; n=10 ; P=0.25)$ or RIT alone $(20 \% ; n=10 ; P=0.25)$. In addition, NFV/RIT, when given 4 hours after SEB/D-gal, was associated with improved survival compared with control $(60 \% ; n=10 ; P<0.05$; Figure $1 C)$. NFV/RIT treatment was also associated with reduced $\mathrm{V}-\beta 8 \mathrm{~T}$ cell apoptosis compared with vehicle control (Figure 1D). 
A

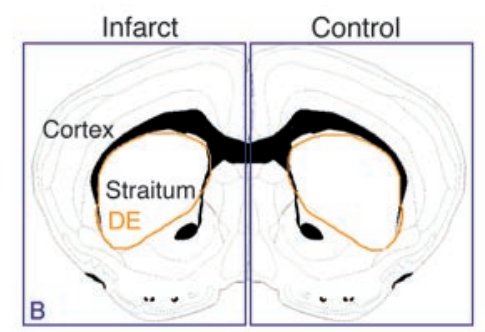

C
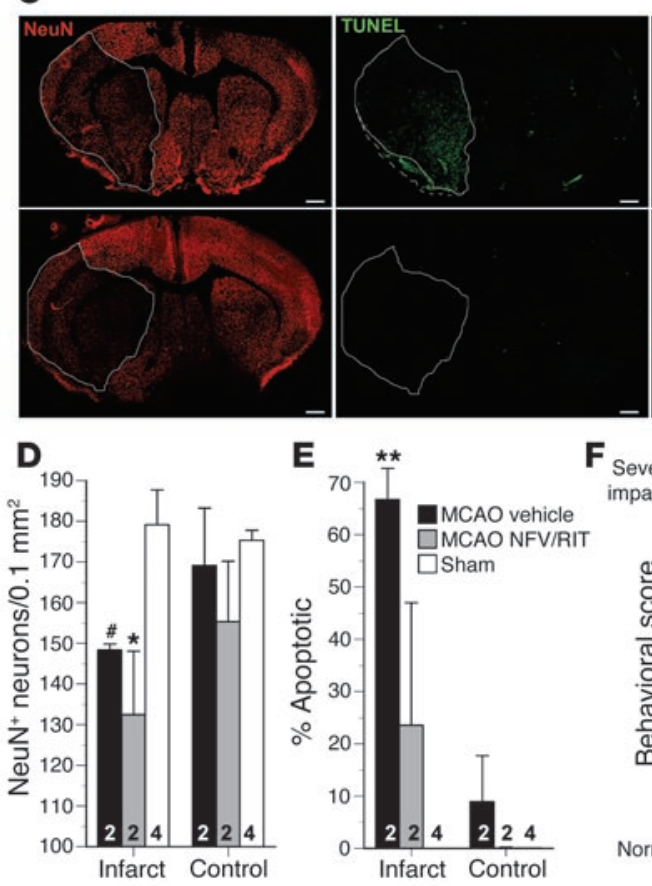

Hemisphere

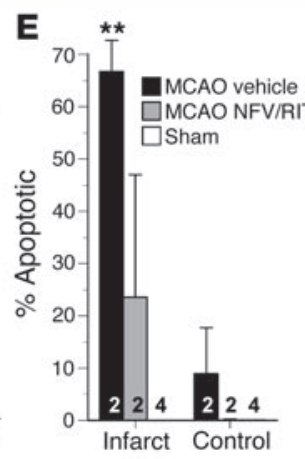

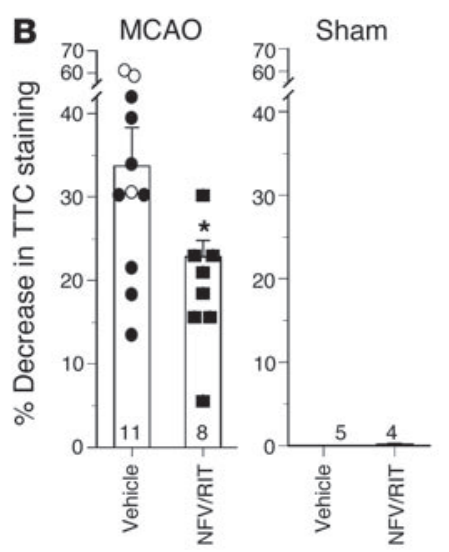

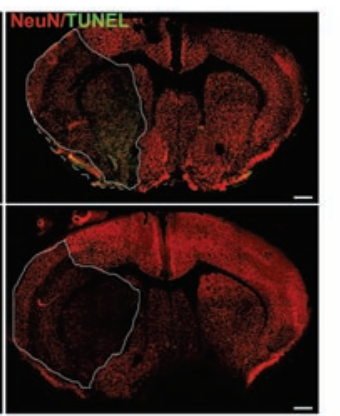

F Severly

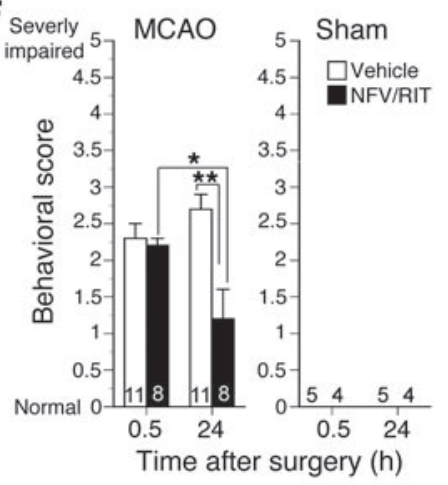

Figure 2

Effects of NFV/RIT on infarct size, neuronal loss, and behavioral impairment following 1 hour of MCAO and 24 hours of reperfusion. (A) Schematic representation of coronal forebrain sections were analyzed. Infarcted and control hemispheres are indicated. DE, diencephaIon. The tissue areas analyzed by TTC as shown in B are indicated in blue. The areas analyzed for NeuN and TUNEL reactivity as shown in $\mathbf{D}$ and $\mathbf{E}$ are indicated in orange. (B) Mice pretreated with NFV/RIT exhibit a smaller infarct 24 hours after surgery, as assessed by TTC staining, relative to mice pretreated with vehicle or control (sham) mice ( ${ }^{\star} P<0.05$, Student's $t$ test). Open circles, no pretreatment; filled circles, vehicle pretreatment; filled squares, NFV/RIT pretreatment. (C) NFV/ RIT protects neurons from apoptotic-like death. (D) Quantitation of overall neuronal loss following MCAO or sham surgery. Both vehicle- and NFV/RIT-treated animals exhibit a comparable reduction in neuronal number following MCAO ( ${ }^{*} P=0.08,{ }^{*} P<0.05$, ANOVA, post-hoc Dunnett $t$ test). (E) NFV/RIT-treated animals are protected from apoptotic-like death following MCAO surgery. The majority of remaining NeuN-positive cells in vehicle-treated mice are apoptotic, while TUNEL reactivity is significantly reduced in NFV/RIT-treated animals ( ${ }^{* *} P<0.01$, ANOVA, post-hoc Dunnett $t$ test). (F) NFV/RIT improves neurological recovery following MCAO. Animals tested immediately after MCAO surgery ( 0.5 hours) show equivalent motor deficits. After 24 hours of reperfusion, NFV/RIT exhibit significant behavioral improvement relative to 0.5 hours or vehicletreated cells. ${ }^{\star} P<0.05 ;{ }^{\star \star} P<0.01$, ANOVA, post-hoc Tukey test. Data represent mean \pm SEM.
Given the substantial reduction in apoptosis and the improvement in survival afforded by NFV/RIT in the Jo- 2 and SEB models, we next assessed the impact of NFV/RIT on cerebral injury in the middle cerebral artery occlusion (MCAO) model of focal ischemia. C57BL/6 mice were pretreated by oral gavage with either NFV/RIT or vehicle ( $2 \%$ EtOH) 24,16 , and 8 hours before sham or MCAO surgery (vehicle control, sham surgery, $n=7$; vehicle control, $\mathrm{MCAO}, n=10 ; \mathrm{NFV} / \mathrm{RIT}$, sham surgery, $n=7 ; \mathrm{NFV} / \mathrm{RIT}$, MCAO surgery, $n=10$ ). A fifth cohort received MCAO surgery without NFV/RIT or vehicle pretreatment $(n=5)$. Infarct size in the striatum and cortex was assessed by 2,3,5-triphenyltetrazolium (TTC) staining (Figure 2A). After 1 hour of MCAO followed by 24 hours of reperfusion, vehicle-treated animals or mice that were not orally gavaged exhibited a $34 \%$ reduction in TTC staining (Figure $2 \mathrm{~B}$ ). Infarct size as measured by TTC staining was $23 \%$ $(P<0.05$ compared with control) in the NFV/RIT treatment group (Figure 2B). We next assessed neuronal number in the striatum by immunodetection of the neuron-specific marker NeuN (24). Both vehicle- and NFV/RIT-treated animals exhibited a comparable, approximately $20 \%$ loss of NeuN-positive neurons after 1 hour of MCAO and 24 hours of reperfusion (Figure 2, C and D). However, NFV/RIT significantly reduced apoptotic-like death, as detected by TUNEL/NeuN double labeling (Figure 2, C and E). In vehicle-treated animals, $67 \%$ of the remaining NeuN-positive neurons in the striatum were actively undergoing DNA fragmentation 24 hours after surgery (Figure 2, C and E); however, only 24\% $(P<0.01)$ of NeuN-positive neurons were TUNEL reactive in NFV/RIT-treated mice (Figure 2, C and E). Finally, we determined whether the neuroprotection afforded by NFV/RIT pretreatment manifested in improved behavioral recovery. Neurological deficits in motor function were assessed using an expanded 6-point scale modified from refs. 25, 26 (see Methods). Animals were tested immediately after 0.5 hours of MCAO and again 24 hours after reperfusion. NFV/RIT-pretreated animals exhibited comparable motor deficits after 1 hour of MCAO compared with vehicle-treated or control animals (no pretreatments) but evident behavioral improvement 24 hours after surgery (Figure 2F), consistent with the reduction in apoptotic loss and reduced infarct size.

To analyze the antiapoptotic mechanism of NFV/RIT in vivo, we compared apoptotic signaling in Jurkat $\mathrm{T}$ cells stimulated in 
A

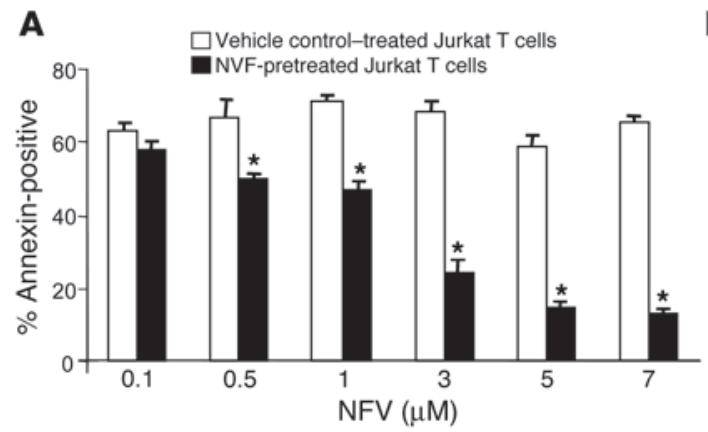

D
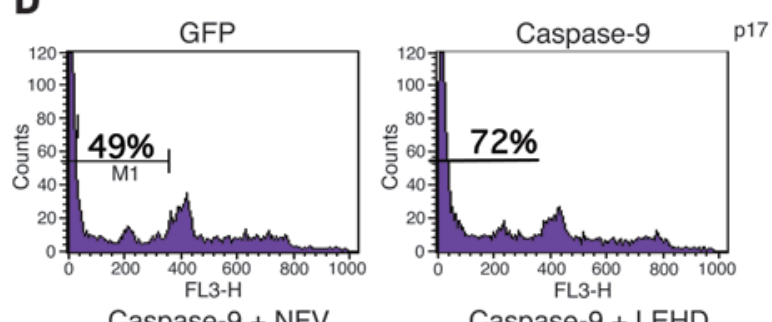

Caspase-9 + LEHD

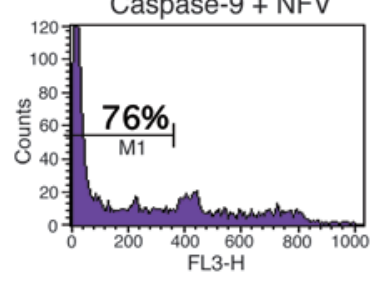

G
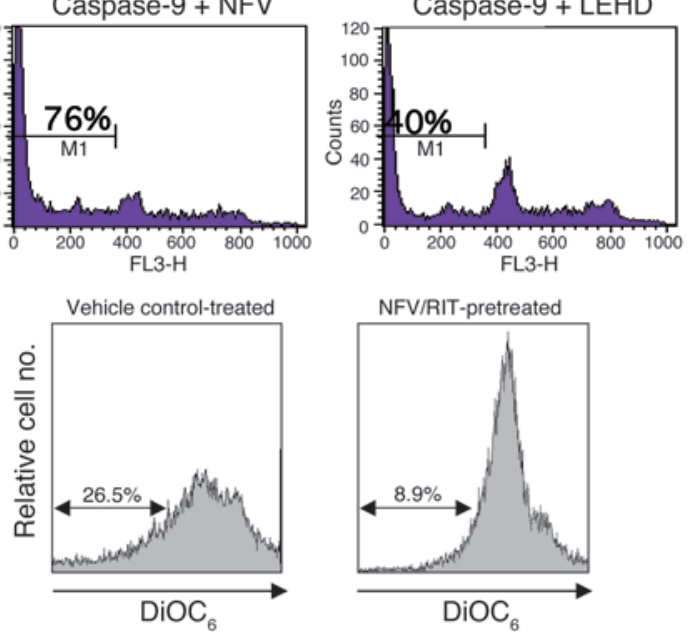

B

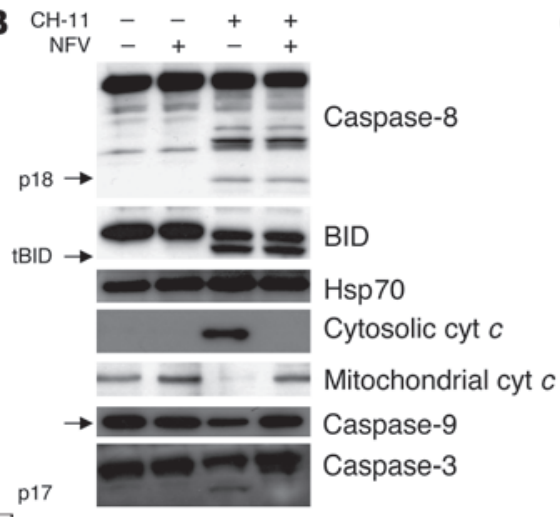

E

Vehicle control-treated

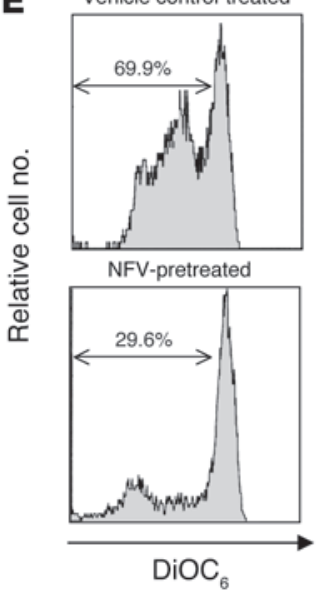

C

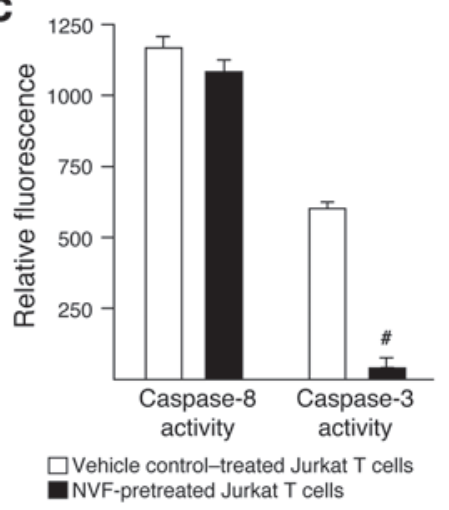

$\mathbf{F}$

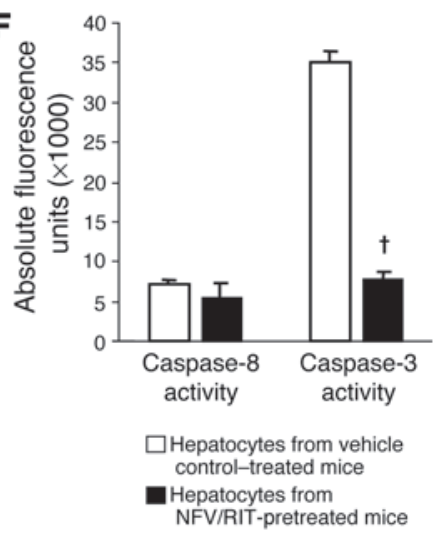

NFV/RIT-pretreated mice

Figure 3

Effect of NFV on apoptotic signaling events in vitro and in vivo. (A) Jurkat T cells were stimulated with agonistic anti-Fas antibody (CH-11) in the presence or absence of varying concentrations of $\mathrm{PI}$ and analyzed for annexin positivity. ${ }^{\star} P<0.05$. (B) Western blot analysis of the Fas signaling events of caspase-8, -9 , and -3 cleavage, BID cleavage, or cytosolic translocation of cytochrome $c$ (cyt $c$ ) in Jurkat T cells treated or not treated with $\mathrm{CH}-11$ with or without NFV. tBID, truncated BID. (C) Caspase-8 and caspase-3 activity was also assessed in Jurkat T cells stimulated with $\mathrm{CH}-11$ in the presence or absence of NFV. ${ }^{\#} P<0.01$. (D) Jurkat cells were transiently transfected by a GFP plasmid or a caspase-9 GFP plasmid, treated or not treated with $7 \mu \mathrm{M}$ NFV or $100 \mu \mathrm{M}$ LEHD, cultured for 6 hours, stained with PI, and analyzed by cytofluorometry for hypoploidy. (E) Jurkat cells stimulated with $\mathrm{CH}-11$ in the presence or absence of NFV were analyzed for loss of DiOC 6 retention. Hepatocytes isolated from mice receiving Jo-2 with NFV/RIT or control (as in Figure 1) were also analyzed for caspase-8 and caspase-3 activity (F) and loss of $\mathrm{DiOC}_{6}$ retention $(\mathbf{G}) .{ }^{\dagger} P<0.005$.

vitro with CH-11 anti-Fas antibody with the apoptotic signaling in hepatocytes isolated from mice treated in vivo with IV Jo-2 antiFas antibody. In vitro pretreatment of Jurkat T cells with NFV followed by stimulation with $\mathrm{CH}-11$ resulted in a dose-dependent reduction in apoptosis (Figure 3A). NFV-pretreated Jurkat T cells had similar caspase-8 cleavage and activity and Bid cleavage as seen in vehicle control cells. However, mitochondrial membrane permeability $\left(\Delta \Psi_{\mathrm{m}}\right)$, cytochrome $c$ release, caspase-9 cleavage, and caspase-3 cleavage and activity were all reduced by NFV pretreatment (Figure 3, B-E), which suggests that NFV inhibition of apoptosis occurs upstream of caspase-9 in the apoptotic pathway. Moreover, transient expression of caspase- 9 in Jurkat $\mathrm{T}$ cells resulted in hypoploidy that was not inhibited by NFV yet was inhibited by the caspase-9 inhibitor, LEHD, which confirms that NFV blocks apoptosis upstream of caspase-9 activation. Similar analyses were performed ex vivo on hepatocytes isolated from mice pretreated with either NFV/RIT or vehicle control and subsequently challenged with IV Jo-2. In these cells, caspase-8 activation was unaltered by NFV/RIT pretreatment, whereas caspase-3 activity was significantly $(P<0.005)$ inhibited (Figure 3F). Additionally, loss of $\Delta \Psi_{\mathrm{m}}$ was evident in vehicle control hepatocytes but was blocked in the hepatocytes of mice that received NFV/RIT pretreatment (Figure $3 \mathrm{G})$. Therefore, in vitro and in vivo, PIs inhibit mitochondrial $\Delta \Psi_{\mathrm{m}}$ and subsequent postmitochondrial signaling events.

To confirm that the effect of NFV localizes to mitochondria, we treated isolated mitochondria with $\mathrm{Vpr}$, atractyloside (ATR), Bax 
and determined that NFV inhibits mitochondrial swelling induced by all 3 stimuli (Figure 4A). Inhibition of Bax-induced apoptosis might indicate a loss of Bax activation and translocation or a lack of effect of Bax on $\Delta \Psi_{\mathrm{m}}$ (reviewed in refs. 27, 28). We therefore directly assessed whether NFV inhibited mitochondrial translocation and activation of Bax. We stimulated cells through the Fas receptor in the presence of NFV or DMSO vehicle control and analyzed them for nuclear morphology using Hoechst 33342 staining and for Bax activation by immunofluorescence using an antibody specific for the activated conformation of Bax. Control (untreated) cells had no evidence of Bax activation and had intact nuclei. Fasstimulated cells exhibited both Bax activation with punctate Bax staining and fragmented nuclei, consistent with apoptosis. NFVpretreated cells showed punctate staining for activated Bax, yet no nuclear fragmentation was observed (Figure 4B).

Furthermore, we induced apoptosis by overexpression of Bax. Bax was cotransfected with GFP, and consequently apoptosis was assessed specifically in the GFP-positive cell populations. NFV treatment inhibited $\Delta \Psi_{\mathrm{m}}$ loss induced by the overexpression of Bax (Figure 4C). Together, therefore, our data indicate that the antiapoptotic action of NFV occurs at the level of mitochondria, downstream of Bax translocation and activation, but invoking mitochondrial $\Delta \Psi_{\mathrm{m}}$.

Mitochondrial loss of $\Delta \Psi_{\mathrm{m}}$ is coincident with the opening of the mitochondrial permeability transition pore complex (PTPC). These events allow the release of cytochrome $c$ into the cytosolic compartment, which, in the presence of dATP, results in the formation of a complex between Apaf- 1 and procaspase- 9 known as the apoptosome. This results in activation of procaspase- $9(29,30)$, which in turn activates the downstream effector caspase-3 (31). A key regulator in this process, therefore, is the mitochondrial PTPC, which is composed of the peripheral benzodiazepine receptor (PBR), adenine nucleotide translocator (ANT), voltage-dependent anion channel (VDAC), as well as other proteins (32). We therefore assessed apoptosis in the presence or absence of NFV in WT yeast or yeast deficient in both isoforms of VDAC or all 3 isoforms of ANT. HIV Vpr initiates apoptosis through binding ANT with affinities in the nanomolar range (33), whereas Vpr does not bind to VDAC in vitro (33). Consistent with prior reports, Vpr was able to induce yeast death only if ANT was present (Figure 5, A and B). Since VDAC and ANT physically interact, and form the principal pore channel of PTPC in a cooperative manner $(34,35)$, absence of VDAC also abrogated the ability of Vpr to cause death (Figure $5 \mathrm{~A}$ ). $\mathrm{H}_{2} \mathrm{O}_{2}$ also induces apoptosis in an ANT-dependent manner, potentially through oxidation of thiol groups within ANT (36-38). Like Vpr, $\mathrm{H}_{2} \mathrm{O}_{2}$ only induced apoptosis if ANT was present, and VDAC was required as well (Figure 5A). Next, we assessed the ability of NFV to inhibit apoptosis in WT yeast following stimulation with $\mathrm{Vpr}$ or $\mathrm{H}_{2} \mathrm{O}_{2}$. WT yeast underwent $\mathrm{Vpr}$ - and $\mathrm{H}_{2} \mathrm{O}_{2}$-induced apoptosis, which was inhibited in a dose- dependent manner by NFV treatment (Figure 5B), arguing that NFV requires the presence of ANT, VDAC, or both.

To further examine the involvement of the components of the PTPC in NFV-mediated inhibition of apoptosis, we next used chemical ligands that selectively interact with different components of the PTPC, causing it to open and resulting in subsequent loss of $\Delta \Psi_{\mathrm{m}}$. PK11195 is an agonist of the $\operatorname{PBR}(39,40), \operatorname{ATR}$ is an agonist of ANT (41), and staurosporine (STS) is an agonist of VDAC $(42,43)$. Jurkat T cells were pretreated with NFV or vehicle control and subsequently treated with these agonists and analyzed for apoptosis. Treatment with PK11195 or STS resulted in apoptosis (Figure 6A), loss of $\Delta \Psi_{\mathrm{m}}$ (Figure 6B), cytochrome $c$ release, caspase- 9 cleavage, caspase- 3 cleavage and activation, and poly (ADP-ribose) polymerase (PARP) cleavage (Figure 6, C and D) that were not altered by NFV, which suggests that NFV does not alter PBR or VDAC-initiated loss of $\Delta \Psi_{\mathrm{m}}$. Repeating these experiments with the ANT-specific agonist ATR resulted in a loss of $\Delta \Psi_{\mathrm{m}}$, cytochrome $c$ release, caspase- 9 cleavage, caspase- 3 cleavage, PARP cleavage, and caspase- 3 activation that were inhibited by NFV (Figure 6, A-D). These differential results suggest that NFV acts as an inhibitor of ANT-dependent PTPC opening.

To confirm that NFV can act specifically with ANT to inhibit its pore function, we used proteoliposomes reconstituted with either entire PTPC or with ANT alone, which release a fluorescent dye following pore opening (44). Consistent with our cellular data, treatment of PTPC liposomes with ATR results in significant fluorescence release that was progressively inhibited by a range $(0.1-10 \mu \mathrm{m})$ of increasing doses of NFV (Figure $7 \mathrm{~A})$. We
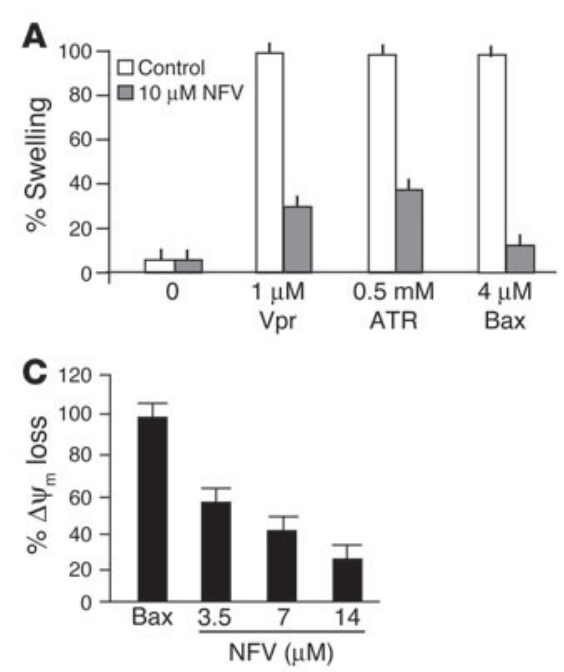

\section{Figure 4}

NFV blocks Bax-induced apoptosis but not Bax activation. (A) Mouse liver mitochondria were incubated with $10 \mu \mathrm{M}$ of NFV followed by $1 \mu \mathrm{M}$ Vpr-derived peptide, $0.5 \mathrm{mM}$ ATR, or $4 \mu \mathrm{M}$ Bax while absorption was assessed at $545 \mathrm{~nm}$. The loss of absorption induced by $0.5 \mathrm{mM}$ ATR within 20 minutes was considered at $100 \%$ of large amplitude swelling. All experiments were reproduced 3 times. (B) Jurkat T cells were treated with an agonistic anti-Fas antibody in the presence or absence of NFV and stained with Hoechst 33342 for nuclear morphology, an antibody (or isotype control) specific for activated Bax, and an Alexa Fluor-conjugated secondary antibody. All cells were stained with Hoechst and varying combinations of NFV or DMSO, $\mathrm{CH}-11$, and anti-Bax or isotype antibody as indicated. (C) Jurkat T cells were transfected with Bax, and immediately following transfection, NFV or control was added and $\Delta \Psi_{\mathrm{m}}$ was assessed. 
A
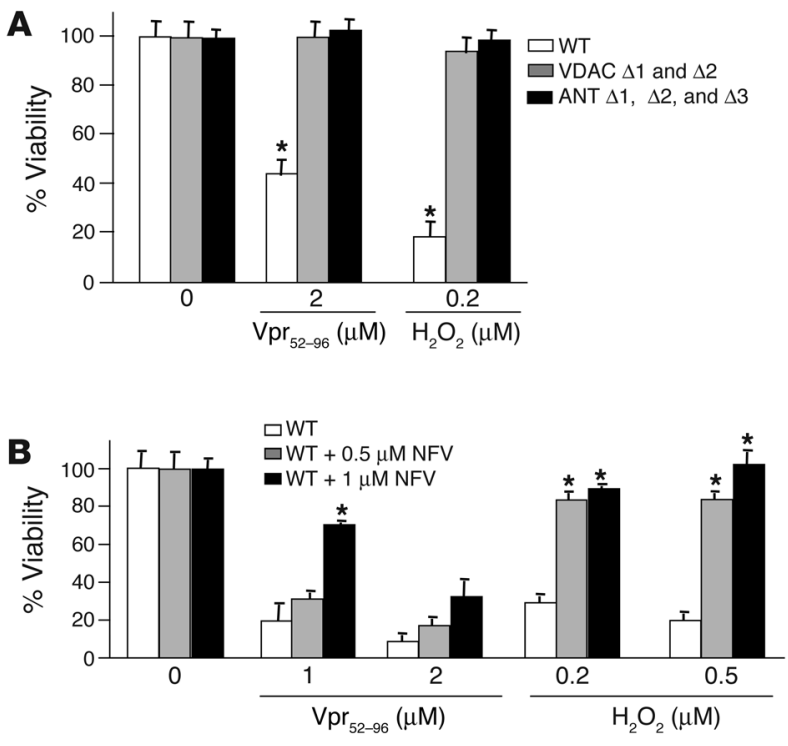

next assessed whether NFV could act directly upon ANT to inhibit its pore function. ANT liposomes were created and treated with 2 distinct ANT ligands, ATR (Figure 7B) and Vpr (Figure 7C). Each ligand resulted in a dose-dependent release of fluorescence from the ANT liposomes that in turn was inhibited $(P<0.05)$ in a doseresponsive manner by NFV.

\section{Discussion}

Here we provide evidence that HIV PIs have the ability to meaningfully impact apoptosis in vivo in a variety of animal models and

\section{Figure 5}

Effects of NFV on apoptosis in yeast. (A) WT yeast or yeast deficient in both isoforms of VDAC (VDAC $\Delta 1$ and $\Delta 2$ ) or 3 isoforms of ANT (ANT $\Delta 1, \Delta 2$, and $\Delta 3$ ) were treated with the apoptosis-inducing agents $\mathrm{Vpr}$ peptide (residues 52-96) or $\mathrm{H}_{2} \mathrm{O}_{2}$ and analyzed for viability. (B) WT yeast treated with varying doses of NFV was treated with the same apoptosis inducers and analyzed for viability. Results are representative of 3 independent experiments. ${ }^{*} P<0.05$.

that this effect localizes to mitochondrial release of proapoptotic factors. Moreover, our in vitro data suggest that NFV acts to inhibit the pore function of the ANT subunit of the mitochondrial PTPC. This effect of NFV permits the premitochondrial signaling events of death receptor-initiated apoptosis yet inhibits mitochondrial loss of $\Delta \Psi_{\mathrm{m}}$ and the subsequent formation of the apoptosome and activation of caspase- 9 and caspase- 3 .

This mechanism of action offers insight into the question of whether cells that have undergone some changes of apoptosis (e.g., caspase- 8 activation) but not others (e.g., $\Delta \Psi_{\mathrm{m}}$ ) will still be fated to death. Hepatocytes from Jo-2-treated mice dosed with NFV/ RIT maintained caspase- 8 activation but not the mitochondrial and postmitochondrial signaling events. Consequently, analysis of these mice affords an opportunity to address the long-term outcome of tissues rescued from death by NFV/RIT treatment. First, since no mortality was observed between days 3 and 30, NFV/RIT treatment prevents rather than delays death. Second, serum AST remains lower in NFV/RIT-treated mice, which suggests maintenance of hepatocyte viability and function. Third, hepatic histology of NFV/RIT-treated mice is preserved at day 30, which demonstrates a lack of delayed tissue damage in mice surviving as a consequence of NFV/RIT therapy. Comparable results were
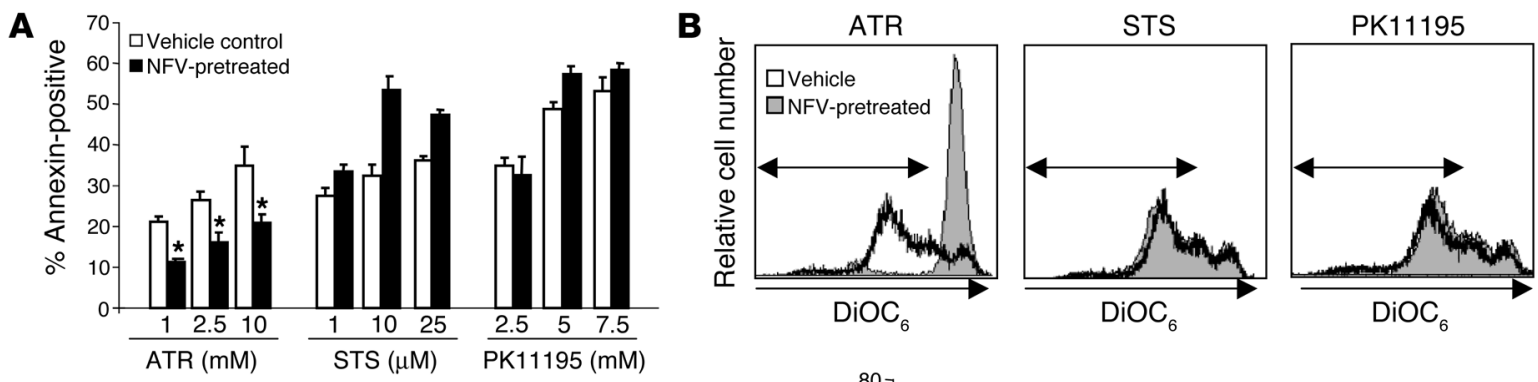

C
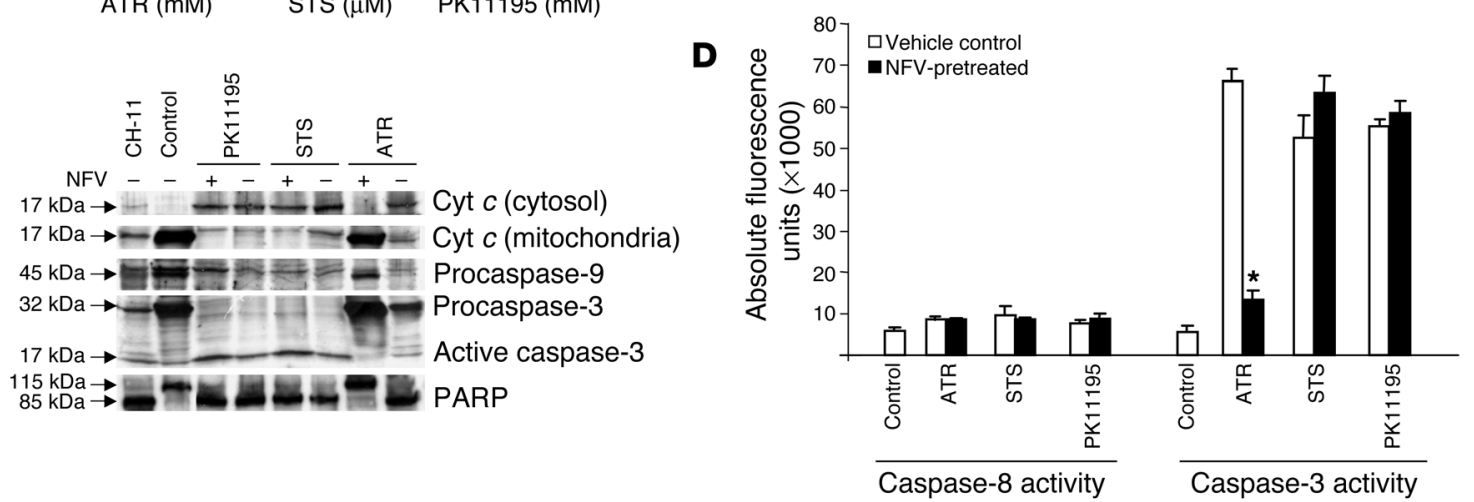

Figure 6

Effects of NFV on the apoptosis-inducing abilities of the selective PTPC agonists. Jurkat T cells were treated with either the ANT agonist ATR, VDAC agonist STS, or PBR agonist PK11195, in the presence or absence of PI, and analyzed for annexin positivity $(\mathbf{A})$, loss of DiOC 6 retention (B) caspase-9, caspase-3, and poly (ADP-ribose) polymerase (PARP) cleavage and cytosolic release of cytochrome $c$ (C), and caspase-8 and caspase-3 activity (D). Results are representative of 3 independent experiments. ${ }^{*} P<0.01$. 
A
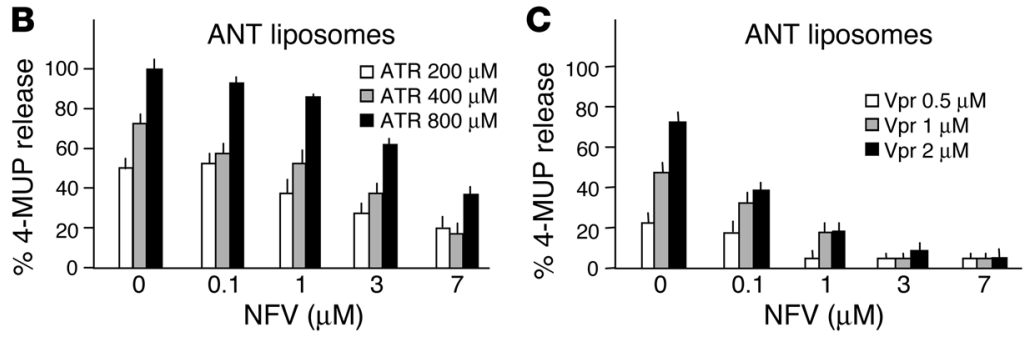

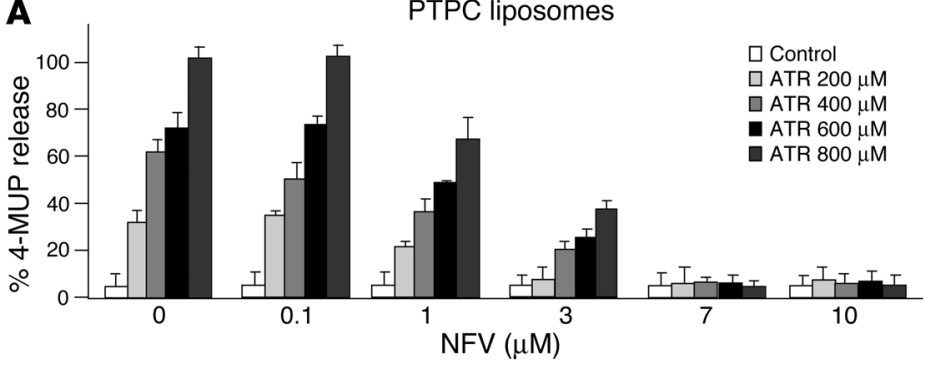

\section{Figure 7}

Effects of NFV on PTPC or ANT pore function. (A) Proteoliposomes containing PTPC complexes were treated with NFV, stimulated with ATR, and analyzed for fluorescence release. Proteoliposomes containing ANT were treated with NFV and analyzed for fluorescence release following stimulation with ATR (B) or Vpr peptide (residues 52-96) (C). Results are representative of 3 independent experiments.

TNF- $\alpha$, PI-treated cells acquire resistance to Fas signaling. It is not clear to what extent adaptive responses involving expression of other mitochondrial carrier proteins (which might assume the functions of ANT) may compensate for the defect in ANT-1 and ANT-2, at either the bioenergetic level or the cell death regulatory level (46). By no means do the data from the knockout model exclude the possibility that pharmacologic apoptosis modulation can be achieved through inhibition of ANT function.

Examining the x-ray crystal structure of ANT, we studied whether NFV can be predicted to interact with observed in the MCAO model of focal ischemia. NFV/RIT did not affect necrotic loss or neuronal damage in the ischemic core, nor did pretreatment improve behavioral indices when animals were tested after recovery from the anesthetic, 30 minutes after the 1 -hour occlusion. However, NFV/RIT significantly reduced subsequent apoptotic-like death observed over the 24-hour reperfusion period, markedly improving neurological scores as a consequence. Therefore, blocking the mitochondrial events of apoptosis represents a viable approach to preventing rather than delaying cell death and a potential means of improving function in pathologic processes involving exaggerated apoptosis.

It is noteworthy that the antiapoptotic effects of NFV/RIT persist even if administered after the apoptosis-initiating event in mice receiving SEB/D-gal, and survival is improved when NFT/ RIT is administered 4 hours later. Although the maximum duration between apoptotic insult and NFV/RIT administration that is still associated with improved outcome remains to be defined, these observations suggest the increased likelihood that such agents might afford clinically useful protection.

Recently, the role of ANT in mitochondrial permeability transition has been questioned. Whereas ANT has been widely regarded as being essential for mitochondrial depolarization and consequent cytochrome $c$ release, apoptosome formation, and apoptosis, a recent report directly challenges that role. Mice were constructed with a complete deficiency in both isoforms of ANT within liver tissues (45). Hepatocytes from these mice had compensatory upregulation of cytochrome $c$ and of cytochrome $c$ oxidase. Mitochondria from these livers contain functional PTPCs, which release cytochrome $c$; however, 3-fold more calcium signal was required to induce the permeability transition than in WT cells. Thus, although the knockout of ANT-1 and ANT-2 genes in hepatocytes does not abolish mitochondrial outer membrane permeabilization induced by calcium in absolute terms, it does significantly reduce the sensitivity of these mitochondria to calcium-induced PTPC opening. Our current data are consistent with some findings discussed in that report; both inhibition of ANT by PIs and genetic absence of ANT result in abrogated mitochondrial response to ANT ligands, including ATR. However, unlike cells from ANT-deficient livers, which remain sensitive to apoptosis induced by Fas ligand and
ANT. Computer simulations of the interactions of NFV with ANT indicated a putative binding site on the matrix side of the transporter protein. Based on this simulation, NFV is predicted to bind close to helices 3 and 5 of ANT (Figure 8). Binding also involves part of the loop connecting helices 3 and 4 (loop M2 in Figure 8), which has been proposed as the ADP binding site (37). Several hydrogen bonds appear to be made between NFV and ANT, in particular with residues K162, G242 (backbone), M239 (backbone), and R243 (Figure 8). Hydrophobic contacts between NFV and ANT constitute the segment around S166 as well as the side chain of R139.

Since multiple apoptotic signals, including death receptor ligation, chemotherapeutics, $\mathrm{BH} 3$-only $\mathrm{Bcl} 2$ family members, UV radiation, and others converge upon mitochondria, drugs that target mitochondrial regulators of apoptosis, such as NFV, are attractive therapeutic candidates. Our data provide the basis for the evaluation of NFV or related compounds as in vivo inhibitors of apoptosis in non-HIV disease states characterized by excessive apoptosis.

\section{Methods}

\section{Mouse treatments}

Mice received either NFV/RIT in $2 \%$ ethanol or vehicle control (2\% ethanol) by oral gavage every 8 hours. Animal treatments were reviewed and approved by the Mayo Foundation Institutional Animal Care and Use Committee, the NRC/IBS Animal Care Committee, and the University of Ottawa Animal Care Committee. Plasma concentrations of NFV and RIT were measured simultaneously using liquid chromatography with tandem mass spectrometry. The lower limit of quantitation for NFV and RIT was $2.5 \mathrm{ng} / \mathrm{ml}$. The accuracy of quality control samples analyzed simultaneously with the study samples ranged from $83.7 \%$ to $107.5 \%$ for NFV and $105.0 \%$ to $116.5 \%$ for RIT. The within-run variability for 3 replicative analyses of the quality control samples was less than $9.7 \%$ for both NFV and RIT. This assay has been cross-validated as part of the International Interlaboratory Quality Control Program for Therapeutic Drug Monitoring in HIV Infection (47). To determine a dosing regimen that would result in relevant plasma concentrations, we treated 6- to 8-week-old BALB/c and C57BL $/ 6$ mice with $250 \mathrm{mg} / \mathrm{kg}$ of NFV (Agouron Pharmaceuticals Inc.) every 8 hours, yet plasma concentrations were undetectable 6 hours after dosing. Since coadministration of RIT in humans significantly increases 


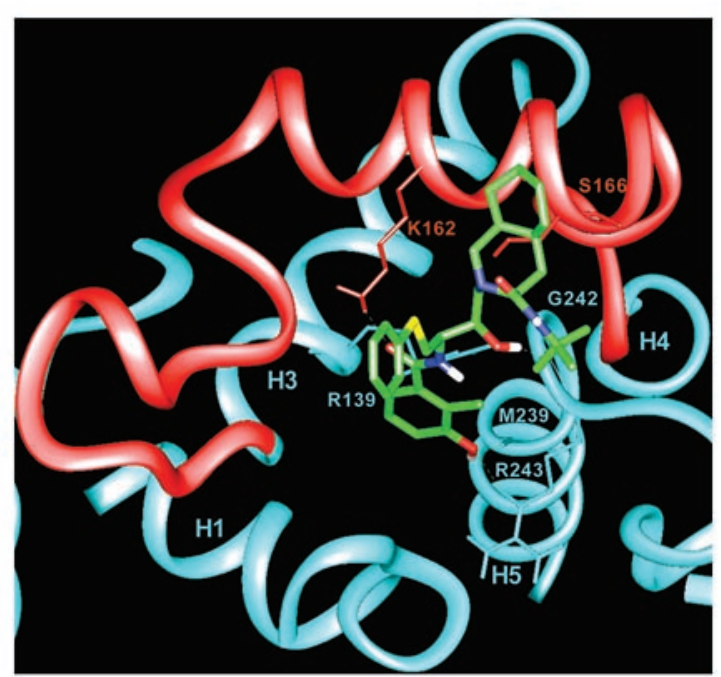

Figure 8

Computer-simulated model of NFV interaction with ANT. Close-up of the proposed binding mode of NFV to the matrix side of ANT. Parts of helices $1,3,4$, and $5(\mathrm{H} 1, \mathrm{H} 3, \mathrm{H} 4$, and $\mathrm{H} 5)$ are shown. Loop M2, connecting helices 3 and 4 , is shown in red. Hydrogen bonds are displayed as dotted lines. Side chains or backbone atoms of selected residues are shown (see Methods). The PI is colored according to atom types: $\mathrm{C}$, green; $\mathrm{O}$, red; $\mathrm{N}$, blue; $\mathrm{S}$, yellow.

NFV levels compared with NFV alone (48), we treated mice every 8 hours with $125 \mathrm{mg} / \mathrm{kg} \mathrm{NFV}$ and $13 \mathrm{mg} / \mathrm{kg}$ RIT (Abbott Laboratories). Eighthour NFV trough levels, ranging from 1,199 to $1,258 \mathrm{ng} / \mathrm{ml}$, were comparable to concentrations observed in HIV-infected patients using NFV. We also assessed whether C57BL/6 mice treated with this dose of NFV/RIT achieved detectable drug levels in the brain. NFV and RIT concentrations in brain tissues were determined after the tissues were washed extensively with PBS, blotted dry with filter paper, placed in $5 \mathrm{ml}$ of $0.1 \mathrm{M}$ phosphoric acid, and homogenized using a polytron. Supernatants were analyzed as described above for drug level determination. Analysis of whole brains revealed levels of NFV between 682 and $986 \mu \mathrm{g} / \mathrm{g}$ of brain tissue. We therefore opted to assess the in vivo antiapoptotic effects of the combination of NFV and RIT using this dosing regimen.

\section{Fas-induced liver failure}

Six-week-old female C57BL/ 6 mice (Charles River Laboratories) received the indicated doses of Jo-2 anti-Fas antibody (BD Biosciences - Pharmingen) by tail vein injection. Moribund animals, or animals who survived until the indicated time points, were sacrificed by $\mathrm{CO}_{2}$ asphyxiation. Clinical chemistries were performed on serum obtained from 3 mice from each treatment group at 4 and 24 hours after Jo- 2 injection. Quantitation of hepatocyte apoptosis by TUNEL assay (Roche Diagnostics Corp.) was averaged from results analyses performed in a blinded manner by 4 reviewers.

\section{SEB-induced shock}

Six-week-old female BALB/c mice (Harlan) received $20 \mathrm{mg}$ D-gal (SigmaAldrich) and $6.5 \mu \mathrm{g}$ SEB (Toxin Technology Inc.) intraperitoneally. Animals were euthanized if they became moribund, laterally recumbent, or hypothermic (rectal temperature $<32^{\circ} \mathrm{C}$ ) at the indicated time points. Spleens were harvested and splenocytes isolated mechanically with plastic mesh. Following red cell lysis and removal of B cells by incubation with nylon wool (1 hour, $37^{\circ} \mathrm{C}$; Accurate Chemical and Scientific Corp.), purified $\mathrm{T}$ cells were stained with monoclonal anti-mouse V- $\beta 8$-FITC antibody (clone F23.1), V-B3-FITC antibody (Clone KJ25), or appropriate isotype control (BD Biosciences - Pharmingen) and then washed with PBS and fixed with $1 \%$ paraformaldehyde. Dual staining with TUNEL-TMR red (Roche Diagnostics Corp.) was performed as per the manufacturer's instructions.

\section{Cerebral ischemia produced by MCAO}

C57BL/6 mice (20-23 g) were obtained from Charles River Laboratories. Under temporary isofluorane anesthesia, mice were subjected mice to MCAO using an intraluminal filament as previously described (49). After 1 hour of MCAO, the filament was withdrawn, blood flow restored to normal by laser Doppler flowmetry, and wounds sutured. Beginning 24 hours before surgery, mice received 3 oral gavages, 8 hours apart, of either NFV/ RIT or vehicle. Following 24 hours of reperfusion, mice were anesthetized with $\mathrm{CO}_{2}$ and sacrificed by either decapitation or lethal injection with sodium pentobarbital and transcardially perfused with $10 \mathrm{mM}$ PBS $(10 \mathrm{mM}$ sodium phosphate and $154 \mathrm{mM} \mathrm{NaCl}$ ) followed by $3.7 \%$ paraformaldehyde in $10 \mathrm{mM}$ PBS. Behavioral impairment following MCAO was assessed at 30 minutes after ischemic surgery when animals had recovered from the anesthetic and 24 hours after reperfusion by 2 investigators blind to the treatment of the mice. A modified 6-point scale was used (25, 26): 0, normal; 1, mild turning behavior with or without inconsistent curling when picked up by tail and less than $50 \%$ attempts to curl to the contralateral side; 2 , mild, consistent curling and more than $50 \%$ attempts to curl to the contralateral side; 3 , strong and immediate consistent curling, mouse holds curled position for more than 1-2 seconds, and mouse's nose almost reaches tail; 4, severe curling progressing into barreling and loss of walking or righting reflex; 5, comatose or moribund. Behavioral scores were averaged within treatment groups for statistical analysis. In unfixed tissue, infarct size was measured by a colorimetric staining method using TTC, as described previously (50). Brains were removed and cut into three 2 -mm-thick coronal slices through forebrain, which were separated into left and right hemispheres and stained with $5 \mathrm{ml}$ of $2 \%$ TTC per hemisphere for 90 minutes at $37^{\circ} \mathrm{C}$. The tissue was rinsed with saline, and the formazan product solubilized in ethanol/DMSO (1:1). After a 24-hour incubation in the dark, the red solvent extracts were diluted 1:20 with fresh ethanol/DMSO solvent in triplicate, absorbance was measured at $485 \mathrm{~nm}$ in a spectrophotometer, and the values were averaged. Percent decrease in brain TTC staining was calculated using the following equation: percent decrease $=[100-($ absorbance of contralateral hemisphere/absorbance of ischemic hemisphere $) \times 100]$; and the values for the ischemic and contralateral sides of the brain in the same animal were compared. Perfused brains were postfixed for 24 hours in $3.7 \%$ paraformaldehyde in $10 \mathrm{mM}$ PBS and cryoprotected in $20 \%$ sucrose solution in $10 \mathrm{mM}$ PBS containing $0.001 \%$ sodium azide. Serial coronal sections (10 $\mu \mathrm{m}$ ) were cryostat cut (Leica Microsystems Inc.). Neuronal apoptosis was confirmed in fixed tissue by reaction for TUNEL (Roche Diagnostics Corp.) and immunolabeling for the neuronal nuclear marker NeuN (Chemicon International) using a Cy3-conjugated anti-mouse IgG secondary antibody (Jackson ImmuoResearch Laboratories Inc.) as described (51). Antibodies were diluted in antibody buffer (10 mM PBS, $0.3 \%$ Triton X-100, and 3\% BSA). We evaluated immunofluorescence using on a DMRXA2 microscope (Leica Microsystems Inc.) equipped for epifluorescence. The number of NeuN-positive/TUNEL-negative cells was counted and tissue area was measured using the Advanced Measurements Module of OpenLab version 3.17 (Improvision) in 2 adjacent sections of both ipsilateral and contralateral striatum and cortex. Means were averaged across treatment groups.

\section{Cell culture and apoptosis induction in vitro}

Jurkat T cells (ATCC) were cultured in RPMI media (Mediatech Inc.), supplemented with $10 \%$ heat-inactivated FBS (Atlanta Biologicals), and peni- 
cillin/streptomycin (Invitrogen Corp.) at $37^{\circ} \mathrm{C}$ in $5 \% \mathrm{CO}_{2}$. Where indicated, cells were incubated with $7 \mu \mathrm{M} \mathrm{NFV}$ or $20 \mu \mathrm{M} z$-VAD-fmk (R\&D Systems) or vehicle (DMSO) overnight prior to induction of apoptosis with indicated doses of CH-11 anti-Fas antibody (Upstate), ATR (Sigma-Aldrich), STS (Calbiochem), or PK11195 (Sigma-Aldrich).

\section{Transfection experiments}

Transient transfection of Jurkat cells with vectors containing a pEGFP gene (pEGFP-N2; BD Biosciences), a Bax-GFP gene (kindly provided by Shigemi Matsuyama, Research Center for Allergy and Immunology, Kanagawa, Japan; ref. 52), and caspase-9-GFP (kindly provided by P. Mehlen, Apoptosis Cancer and Development Laboratory, Lyon, France; ref. 53). Cells were cultured in RPMI 1640, 10\% FBS, and 1\% L-glutamine without antibiotics. Two days before transfection, cells were passed at $1.5 \times 10^{5}$ cells $/ \mathrm{ml}$. For electroporation, $1 \times 10^{7}$ cells were pelleted, resuspended in $250 \mu \mathrm{l}$ of $10 \%$ FBS-RPMI 1640, mixed with $250 \mu \mathrm{l}$ of $20 \mu \mathrm{g}$ DNA, and incubated for 10 minutes at room temperature (RT). The DNA/cell suspension was transferred to a 4-mm cuvette, and electroporation was performed at 10 $\mathrm{ms} / 325 \mathrm{~V}$ using a square wave electroporator (Bio-Rad Laboratories). After 10 minutes at RT, cells were diluted to $20 \mathrm{ml}$ in complete medium without antibiotics and cultured at $37^{\circ} \mathrm{C}$ under $5 \% \mathrm{CO}_{2}$. NVF, DEVD (Bachem), and LEHD (Bachem) were added following electroporation (54).

\section{Hepatocyte isolation}

Four hours after treatment with $7.5 \mu \mathrm{g}$ of Jo-2 antibody, mice were euthanized with pentobarbital (60 mg/kg; Abbott Laboratories) and hepatocytes isolated via a 2 -step collagenase digestion (55). Following red cell lysis, hepatocytes were enumerated and evaluated for apoptosis through loss of $\Delta \Psi_{\mathrm{m}}$ and caspase- 8 and caspase- 3 activity.

\section{Assays of apoptotic signaling: TUNEL}

Briefly, $1 \times 10^{6}$ cells were fixed with $2 \%$ paraformaldehyde, permeabilized with $0.1 \%$ Triton X-100 and $0.1 \%$ citrate, and stained with TUNEL as per the manufacturer's directions (Roche Diagnostics Corp.).

\section{DNA content}

For cell cycle analysis, cells $\left(5 \times 10^{5} / \mathrm{ml}\right)$ were spun down and fixed in $70 \%$ icecold ethanol/PBS, added dropwise while vortexing, kept at $-20^{\circ} \mathrm{C}$, and centrifuged. Cells were resuspended in $1 \mathrm{ml}$ PBS containing RNAse and $50 \mu \mathrm{g} / \mathrm{ml}$ propidium iodide (Invitrogen Corp.) and analyzed in a cytofluorometer (56).

Annexin $V$ and propidium iodide staining. Fifty microliters of annexin binding buffer (BD Pharmingen) was added to $1 \times 10^{6}$ cells in $500 \mu \mathrm{l}$ of media followed by $2 \mu \mathrm{l}$ of annexin V-FITC (BD Pharmingen) and $1 \mu \mathrm{l}$ of propidium iodide (Sigma-Aldrich). The mixture was vortexed and incubated for 30 minutes at $37^{\circ} \mathrm{C}$ and then subjected to FACS analysis.

Western blot analysis. We subjected $40-60 \mu \mathrm{g}$ of protein from whole cell lysates to SDS-PAGE. Following transfer, PVDF membranes (Millipore) were probed with antibodies to caspase- $3(1 \mu \mathrm{g} / \mathrm{ml}$; Gene Therapy Systems Inc.), caspase-9 (1 $\mu \mathrm{g} / \mathrm{ml}$; Medical \& Biological Laboratories Co.), cytochrome $c(1 \mu \mathrm{g} / \mathrm{ml}$; BD Biosciences - Pharmingen), or PARP $(1 \mu \mathrm{g} / \mathrm{ml}$; $\mathrm{BD}$ Biosciences - Pharmingen). Following incubation with goat antimouse HRP (1:10,000; Amersham Biosciences), an enhanced chemiluminescence assay (Amersham Biosciences) was used to detect the proteins of interest. Where indicated, mitochondria were separated from cytosols for subcellular detection of cytochrome $c$, as previously described (57). Briefly, cell lysates were harvested and centrifuged twice at $15,000 \mathrm{~g}\left(4^{\circ} \mathrm{C}\right)$ for 15 minutes to fractionate the cytosolic (supernatant) fraction from the mitochondrial pellet. Aliquots of cytosolic or mitochondrial protein (200 $\mu \mathrm{g}$ ) were separated by $4-15 \%$ gradient SDS-PAGE and probed with monoclonal antibody against cytochrome $c$.
Fluorometric caspase-8 and caspase-3 activity. We resuspended $150-\mu \mathrm{g}$ aliquots of whole cell lysates in reaction buffer with the appropriate fluorogenic caspase substrate (IETD for caspase-8 and DEVD for caspase-3) (R\&D Systems). Activity was determined with a fluorescence plate reader (BIOTEK Inc.) at an excitation wavelength of $400 \mathrm{~nm}$ and emission of $505 \mathrm{~nm}$.

$\mathrm{DiOC}_{6}$ staining. We incubated $1 \times 10^{6}$ intact cells with $40 \mathrm{nM} \mathrm{DiOC} 6$ (Invitrogen Corp.) for 30 minutes at $37^{\circ} \mathrm{C}$ prior to $\mathrm{FACS}$ analysis.

\section{Mitochondrial swelling}

Mitochondria were purified from BALB/c mouse livers on a Percoll gradient (58) and were stored on ice for up to 4 hours. For determination of large amplitude swelling, $5 \mu \mathrm{l}$ of mitochondria $(0.5 \mathrm{mg}$ protein $/ \mathrm{ml})$ was added to $200 \mu \mathrm{l}$ of swelling buffer [0.2 M saccharose, $10 \mathrm{mM}$ TRIS-3-(N-morpholino)-propanesulfonic acid, $5 \mathrm{mM}$ propidium iodide, $1 \mathrm{mM}$ EGTA, 0.35 $\mathrm{mM}$ rotenone, $\mathrm{pH}$ 7.4], and light absorption was recorded for 20 minutes at $545 \mathrm{~nm}$ in a microtiter plate (TECAN GENios). The loss of absorption induced by $0.5 \mathrm{mM}$ ATR was used as a positive control to define maximal large amplitude swelling. For inhibition experiments, NFV or control was always added before the swelling inducers, Vpr-derived peptide, and ATR.

\section{Yeast strains and clonogenic assays}

We pretreated $10^{4}$ cells $/ \mathrm{ml}$ of Saccharomyces cerevisiae M 22-2-1 (genotype MATa ade2 lew2 lys2 his4 trpl ura3 Canr, por1:LEU2, por2:TRP1; gift from M. Forte, Vollum Institute, Portland, Oregon, USA) (59, 60), S. cerevisiae W3011B control strain (MATa ade2, lew2, his3, trpl, ura3, can1), and JL1-3 (genotype like W301-1B, aac1:LEU2 aac2:HIS3, aac3:URA; gift from T. Drgon, NIH, Bethesda, Maryland, USA) (61), with NFV, which was followed by treatment with a Vpr-derived peptide (Genemed Synthesis Inc.) as described (62) or $\mathrm{H}_{2} \mathrm{O}_{2}\left(1\right.$ hour, $\left.28^{\circ} \mathrm{C}\right)$. This was followed by plating on standard YPD agarose medium (200 yeasts/plate) and quantification of the percentage of surviving clones after 48 hours of culture at $28^{\circ} \mathrm{C}$.

\section{Liposome technology}

PTPC $(1 \mathrm{mg} / \mathrm{ml})$ was separated from rat brains and ANT $(0.1 \mathrm{mg} / \mathrm{ml})$ from rat heart mitochondria as previously described $(44,63)$. Immediately after purification, pure proteins were reconstituted into proteoliposomes (phosphatidylcholine/cholesterol [5:1; wt/wt] for PTPC and phosphatidylcholine/ cardiolipin [45:1; wt/wt] for ANT) (64). After extensive dialysis to eliminate the surfactants, proteoliposomes were loaded with 4-methyllumbelliferylphosphate (4-MUP) in $10 \mathrm{mM} \mathrm{KCl}, 10 \mathrm{mM}$ HEPES, $125 \mathrm{mM}$ saccharose ( $\mathrm{pH}$ 7.4), by sonication (25\%, 22 seconds on ice) using a Misonix 550 sonicator (Misonix Inc.), washed on Sephadex PD-10 columns (Amersham Biosciences) and dispensed in 96-well microtiter plates (64). We incubated $25 \mu \mathrm{l}$ of proteoliposomes with the indicated agents (30 minutes for NFV and 60 minutes for ATR and $\mathrm{Vpr}_{52-96}$ at RT). External 4-MUP was then converted to 4-methylumbelliferone (4-MU) by the addition of alkaline phosphatase in the presence of $\mathrm{MgCl}_{2}$ for 15 minutes at $37^{\circ} \mathrm{C}$. The release of 4-MU was measured by spectrofluorometry (excitation $360 \mathrm{~nm}$, emission $450 \mathrm{~nm}$ ). The maximum 4-MUP release was determined by adding $5 \%$ Triton X-100 to proteoliposomes. The percentage of 4-MUP release induced by treatment of liposomes by ATR was determined as [(ATR - treated liposome fluorescence - untreated liposome fluorescence)/(TX-100-treated liposome fluorescence - untreated liposomes fluorescence) $] \times 100$. The maximal fluorescence induced by $800 \mu \mathrm{M}$ ATR was then identified as 100\% 4-MUP release, and the fluorescence induced by the treatment of liposomes by another product or another dose was calculated as a percentage of ATR-induced 4-MUP release.

\section{Activated Bax immunofluorescence}

Jurkat T cells were treated overnight with NFV or vehicle control before being induced to undergo apoptosis with $0.3 \mu \mathrm{g} / \mathrm{ml}$ of CH-11. Teflon- 
coated 10-well immunofluorescence slides (Fischer Scientific International) were pretreated with poly-L-lysine (Polysciences Inc.) for 1 hour at RT, washed with water, and allowed to air dry. Fifty microliters of a $0.5 \times 10^{6}$ cell suspension was aliquoted to each well and allowed to adhere for 1 hour at $37^{\circ} \mathrm{C}$. Following 1 wash with PBS, cells were fixed with $2 \%$ paraformaldehyde and washed again with PBS. Cells were then treated with 1:250 of mouse anti-Bax (clone 6A7; BD Biosciences - Pharmingen) or mouse isotype in antibody (Santa Cruz Biotechnology Inc.) in PBS with $1 \mathrm{mg} / \mathrm{ml} \mathrm{BSA}$ and $100 \mu \mathrm{g} / \mathrm{ml}$ digitonin for 1 hour at RT, followed by 2 washes with PBS. Cells were then incubated for an additional hour at RT in 1:500 of goat anti-mouse Alexa Fluor 633 (Invitrogen Corp.) and $1 \mathrm{mg} /$ $\mathrm{ml}$ Hoechst 33342 (Invitrogen Corp.) in PBS with $1 \mathrm{mg} / \mathrm{ml} \mathrm{BSA}$ and 100 $\mu \mathrm{g} / \mathrm{ml}$ digitonin. Cells then underwent a final wash, were coverslipped, and imaged with laser scanning confocal microscopy (LSM510; Zeiss) at an absorption/emission wavelength of $632 / 647 \mathrm{~nm}$ and $340 / 450 \mathrm{~nm}$ for Alexa Fluor 633 and Hoechst 33342, respectively.

\section{Computer modeling}

Docking of NFV to ANT was carried out with the program QXP (65), using the FULLDOCK+ algorithm implemented in the 2003 version of the program. The recently published crystal structure of ANT (66) was used as the target structure. During docking, both NFV and the protein side chains were treated as totally flexible, and the backbone atoms of the protein were allowed to move under constraints.

\section{Statistics}

Data were analyzed using the Student's $t$ test for normally distributed data, ANOVA for binary variables, and the post hoc Tukey test for assessing multiple treatment groups.

\section{Acknowledgments}

A.D. Badley is supported by grants from the NIH (R01 AI6226101-1 and R01 AI40384) and the Burroughs Wellcome Award for Translational Research (ID\#1005160). S.A.L. Bennett is supported by grants from Alzheimer Society of Canada, the Canadian Institutes of Health Research (CIHR), and the Alzheimer Society of Saskatchewan. S.A.L. Bennett is a CIHR New Investigator and an Ontario Mental Health Foundation Intermediate Investigator. G. Kroemer is supported by grants from Agence Nationale de Recherché sur le Sida and Sidaction. S.T. Hou is supported by grants from the Heart and Stroke Foundation of Canada. C. Brenner is supported by grants from the Foundation pour la Recherche Medical, the French Ministry of Science, the Centre National pour la Recherche Scientifique, and the Association pour la Recherche sur le Cancer. A. Tarze received a fellowship from the French Ministry of Science. T.C. Moffat received an Alzheimer Society of Canada/CIHR doctoral fellowship. The authors gratefully acknowledge the administrative expertise of A. Carisse and T. Hoff and the technical assistance of A. Desbois and offer special thanks to S. Kilborn and J. Keyte for veterinary assistance and advice.

Received for publication August 5, 2004, and accepted in revised form April 14, 2005.

Address correspondence to: Andrew D. Badley, Division of Infectious Diseases, Mayo Clinic College of Medicine, 200 First Street NW, Rochester, Minnesota 55905, USA. Telephone: (507) 2843747; Fax: (507) 284-3757; E-mail: badley.andrew@mayo.edu.
1. Phenix, B.N., Cooper, C., Owen, C., and Badley, A.D. 2002. Modulation of apoptosis by HIV protease inhibitors. Apoptosis. 7:295-312.

2. Zhong, D.S., et al. 2002. HIV protease inhibitor ritonavir induces cytotoxicity of human endothelial cells. Arterioscler. Thromb. Vasc. Biol. 22:1560-1566.

3. Gaedicke, S., et al. 2002. Antitumor effect of the human immunodeficiency virus protease inhibitor ritonavir: induction of tumor-cell apoptosis associated with perturbation of proteasomal proteolysis. Cancer Res. 62:6901-6908.

4. Pajonk, F., Himmelsbach, J., Riess, K., Sommer, A., and McBride, W.H. 2002. The human immunodeficiency virus (HIV)-1 protease inhibitor saquinavir inhibits proteasome function and causes apoptosis and radiosensitization in non-HIV-associated human cancer cells. Cancer Res. 62:5230-5235.

5. Estaquier, J., et al. 2002. Effects of antiretroviral drugs on human immunodeficiency virus type 1induced CD4(+) T-cell death. J. Virol. 76:5966-5973.

6. Sloand, E.M., Young, N.S., Sato, T., Kim, S., and Maciejewski, J.P. 1998. Inhibition of interleukin1beta-converting enzyme in human hematopoietic progenitor cells results in blockade of cytokinemediated apoptosis and expansion of their proliferative potential. Exp. Hematol. 26:1093-1099.

7. Weichold, F.F., et al. 1999. HIV-1 protease inhibitor ritonavir modulates susceptibility to apoptosis of uninfected T cells. J. Hum. Virol. 2:261-269.

8. Sloand, E.M., et al. 1999. Human immunodeficiency virus type 1 protease inhibitor modulates activation of peripheral blood CD4(+) T cells and decreases their susceptibility to apoptosis in vitro and in vivo. Blood. 94:1021-1027.

9. Sloand, E.M., et al. 2000. Protease inhibitors stimulate hematopoiesis and decrease apoptosis and ICE expression in CD34(+) cells. Blood. 96:2735-2739.

10. Ghibelli, L., et al. 2003. Anti-apoptotic effect of HIV protease inhibitors via direct inhibition of calpain.
Biochem. Pharmacol. 66:1505-1512.

11. Phenix, B.N., Lum, J.J., Nie, Z., Sanchez-Dardon, J., and Badley, A.D. 2001. Anti-apoptotic mechanism of HIV protease inhibitors: preventing mitochondrial transmembrane potential loss. Blood. 98:1078-1085.

12. Matarrese, P., et al. 2003. Mitochondrial membrane hyperpolarization hijacks activated $T$ lymphocytes toward the apoptotic-prone phenotype: homeostatic mechanisms of HIV protease inhibitors. J. Immunol. 170:6006-6015.

13. Barreiro, P., Soriano, V., Casas, E., and GonzalezLahoz, J. 2002. Different degree of immune recovery using antiretroviral regimens with protease inhibitors or non-nucleosides. AIDS. 16:245-249.

14. Benito, J.M., et al. 2002. Differences in cellular activation and apoptosis in HIV-infected patients receiving protease inhibitors or nonnucleoside reverse transcriptase inhibitors. AIDS Res. Hum. Retroviruses. 18:1379-1388.

15. Kravcik, S., et al. 2001. Comparative CD4 T-cell responses of reverse transcriptase inhibitor therapy with or without nelfinavir matched for viral exposure. HIV Clin. Trials. 2:160-170.

16. Landay, A.L., et al. 2003. Immune reconstitution is comparable in antiretroviral-naive subjects after 1 year of successful therapy with a nucleoside reverse-transcriptase inhibitor- or protease inhibitor-containing antiretroviral regimen. J. Infect. Dis. 188:1444-1454.

17. Zeldin, R.K., and Petruschke, R.A. 2004. Pharmacological and therapeutic properties of ritonavirboosted protease inhibitor therapy in HIV-infected patients. J. Antimicrob. Chemother. 53:4-9.

18. Zender, L., et al. 2003. Caspase 8 small interfering RNA prevents acute liver failure in mice. Proc. Natl. Acad. Sci. U. S. A. 100:7797-7802.

19. Song, E., et al. 2003. RNA interference targeting Fas protects mice from fulminant hepatitis. Nat.
Med. 9:347-351.

20. Kovalovich, K., et al. 2001. Interleukin-6 protects against Fas-mediated death by establishing a critical level of anti-apoptotic hepatic proteins FLIP, Bcl-2, and Bcl-xL. J. Biol. Chem. 276:26605-26613.

21. Jiang, H., et al. 1995. Murine CD8+ T cells that specifically delete autologous CD4+ T cells expressing $\mathrm{V}$ beta 8 TCR: a role of the Qa-1 molecule. Immunity. 2:185-194

22. Miethke, T., et al. 1992. T cell-mediated lethal shock triggered in mice by the superantigen staphylococcal enterotoxin B: critical role of tumor necrosis factor. J. Exp. Med. 175:91-98.

23. Aoki, Y., et al. 1995. Protective effect of granulocyte colony-stimulating factor against T-cell-meditated lethal shock triggered by superantigens. Blood. 86:1420-1427.

24. Mullen, R.J., Buck, C.R., and Smith, A.M. 1992. NeuN, a neuronal specific nuclear protein in vertebrates. Development. 116:201-211.

25. Zausinger, S., Hungerhuber, E., Baethmann, A., Reulen, H., and Schmid-Elsaesser, R. 2000. Neurological impairment in rats after transient middle cerebral artery occlusion: a comparative study under various treatment paradigms. Brain Res. 863:94-105.

26. Frenkel, D., et al. 2003. Nasal vaccination with myelin oligodendrocyte glycoprotein reduces stroke size by inducing IL-10-producing CD4+ T cells. J. Immunol. 171:6549-6555.

27. Martinou, J.-C., and Green, D.R. 2001. Breaking the mitochondrial barrier. Nat. Rev. Mol. Cell Biol. 2:63-67.

28. Zamzami, N., and Kroemer, G. 2001. The mitochondrion in apoptosis: how Pandora's box opens. Nat. Rev. Mol. Cell Biol. 2:67-71.

29. Zou, H., Hanzel, W.J., Liu, X., Lutschg, A., and Wang, X. 1997. Apaf-1, a human protein homologous to C. elegans CED-4, participates in cytochrome c-dependent activation of caspase-3. Cell. 90:405-413. 
30. Zou, H., Li, Y., Liu, X., and Wang, X. 1999. An APAF-1 cytochrome c multimeric complex is a functional apoptosome that activates procaspase-9. J. Biol. Chem. 274:11549-11556.

31. Srinivasula, S.M., Ahmad, M., Fernandes-Alnemri, T., and Alnemri, E.S. 1998. Autoactivation of procaspase- 9 by Apaf-1-mediated oligomerization. Mol. Cell. 1:949-957.

32. Vieira, H.L.A., et al. 2000. Permeabilization of the mitochondrial inner membrane during apoptosis: impact of the adenine nucleotide translocator. Cell Death Differ. 7:1146-1154.

33. Jacotot, E., et al. 2001. Control of mitochondrial membrane permeabilization by adenine nucleotide translocator interacting with HIV-1 viral protein rR and Bcl-2. J. Exp. Med. 193:509-519.

34. Halestrap, A.P., and Brenner, C. 2003. The adenine nucleotide translocase: a central component of the mitochondrial permeability transition pore and key player in cell death. Curr. Med. Chem. 10:1507-1525.

35. Verrier, F., et al. 2004. Dynamic evolution of the adenine nucleotide translocase interactome during chemotherapy-induced apoptosis. Oncogene. 23:8049-8064.

36. Kanno, T., et al. 2004. Oxidative stress underlies the mechanisms for $\mathrm{Ca} 2+$-induced permeability transition of mitochondria. Free Radic. Res. 38:27-35.

37. Halestrap, A.P., Woodfield, K.Y., and Connern, C.P. 1997. Oxidative stress, thiol reagents, and membrane potential modulate the mitochondrial permeability transition by affecting nucleotide binding to the adenine nucleotide translocase. J. Biol. Chem. 272:3346-3354.

38. Nakagawa, Y. 2004. Initiation of apoptotic signal by the peroxidation of cardiolipin of mitochondria. Ann. N. Y. Acad. Sci. 1011:177-184.

39. Decaudin, D., et al. 2002. Peripheral benzodiazepine receptor ligands reverse apoptosis resistance of cancer cells in vitro and in vivo. Cancer Res. 62:1388-1393

40. Hirsch, T., et al. 1998. PK11195, a ligand of the mitochondrial benzodiazepine receptor, facilitates the induction of apoptosis and reverses Bcl-2-mediated cytoprotection. Exp. Cell Res. 241:426-434.

41. Haouzi, D., et al. 2002. Mitochondrial permeability transition as a novel principle of hepatorenal toxicity in vivo. Apoptosis. 7:395-405.
42. Duan, S., et al. 2003. Mitochondrial outer membrane permeability change and hypersensitivity to digitonin early in staurosporine-induced apoptosis. J. Biol. Chem. 278:1346-1353.

43. Cheng, E.H.-Y., Sheiko, T.V., Fisher, J.K., Craigen, W.J., and Korsmeyer, S.J. 2003. VDAC2 inhibits BAK activation and mitochondrial apoptosis. Science. 301:513-517.

44. Brenner, C., Marzo, I., de Araujo Vieira, H.L., and Kroemer, G. 2000. Purification and liposomal reconstitution of permeability transition pore complex. Methods Enzymol. 322:243-252.

45. Kokoszka, J.E., et al. 2004. The ADP/ATP translocator is not essential for the mitochondrial permeability transition pore. Nature. 427:461-465.

46. Halestrap, A.P. 2004. Mitochondrial permeability: dual role for the ADP/ATP translocator? Nature 430:984.

47. Aarnoutse, R.E., et al. 2002. International interlaboratory quality control program for measurement of antiretroviral drugs in plasma. Antimicrob. Agents Chemother. 46:884-886.

48. Raines, C.P., et al. 2000. Safety, tolerability, and antiretroviral effects of ritonavir- nelfinavir combination therapy administered for 48 weeks. J. Acquir Immune Defic. Syndr. 25:322-328.

49. MacManus, J.P., et al. 2003. Absence of the transcription factor E2F1 attenuates brain injury and improves behavior after focal ischemia in mice. J. Cereb. Blood Flow Metab. 23:1020-1028.

50. Preston, E., and Webster, J. 2000. Spectrophotometric measurement of experimental brain injury. J. Neurosci. Methods. 94:187-192.

51. Melanson-Drapeau, L., et al. 2003. Oligodendrocyte progenitor enrichment in the connexin 32 null-mutant mouse. J. Neurosci. 23:1759-1768.

52. Sawada, M., et al. 2003. Ku70 suppresses the apoptotic translocation of Bax to mitochondria. Nat. Cell Biol. 5:320-329.

53. Forcet, C., et al. 2001. The dependence receptor DCC (deleted in colorectal cancer) defines an alternative mechanism for caspase activation. Proc. Natl. Acad. Sci. U. S. A. 98:3416-3421.

54. Trushin, S.A., et al. 2003. Protein kinase Calpha (PKCalpha) acts upstream of PKCtheta to activate IkappaB kinase and NF-kappaB in T lymphocytes. Mol. Cell. Biol. 23:7068-7081.
55. Seglen, P.O. 1976. Preparation of isolated rat liver cells. Methods Cell Biol. 13:29-83.

56. Zamzami, N., et al. 1995. Reduction in mitochondrial potential constitutes an early irreversible step of programmed lymphocyte death in vivo. J. Exp. Med. 181:1661-1672.

57. Nie, Z., et al. 2002. HIV-1 protease processes procaspase 8 to cause mitochondrial release of cytochrome c, caspase cleavage and nuclear fragmentation. Cell Death Differ. 9:1172-1184.

58. Susin, S.A., Larochette, N., Geuskens, M., and Kroemer, G. 2000. Purification of mitochondria for apoptosis assays. Methods Enzymol. 322:205-208.

59. Blachly-Dyson, E., et al. 1993. Cloning and functional expression in yeast of two human isoforms of the outer mitochondrial membrane channel, the voltage-dependent anion channel. J. Biol. Chem. 268:1835-1841.

60. Blachly-Dyson, E., Song, J., Wolfgang, W.J., Colombini, M., and Forte, M. 1997. Multicopy suppressors of phenotypes resulting from the absence of yeast VDAC encode a VDAC-like protein. Mol. Cell. Biol. 17:5727-5738.

61. Drgon, T., Sabova, L., Nelson, N., and Kolarov, J. 1991. ADP/ATP translocator is essential only for anaerobic growth of yeast Saccharomyces cerevisiae. FEBS Lett. 289:159-162.

62. Macreadie, I.G., Arunagiri, C.K., Hewish, D.R., White, J.F., and Azad, A.A. 1996. Extracellular addition of a domain of HIV-1 Vpr containing the amino acid sequence motif $\mathrm{H}(\mathrm{S} / \mathrm{F}) \mathrm{RIG}$ causes cell membrane permeabilization and death. Mol. Microbiol. 19:1185-1192.

63. Marzo, I., Brenner, C., and Kroemer, G. 1998. The central role of the mitochondrial megachannel in apoptosis: evidence obtained with intact cells, isolated mitochondria, and purified protein complexes. Biomed. Pharmacother. 52:248-251.

64. Belzacq, A.S., et al. 2003. Bcl-2 and Bax modulate adenine nucleotide translocase activity. Cancer Res. 63:541-546.

65. McMartin, C., and Bohacek, R.S. 1997. QXP: powerful, rapid computer algorithms for structure-based drug design. J. Comput. Aided Mol. Des. 11:333-344.

66. Pebay-Peyroula, E., et al. 2003. Structure of mitochondrial ADP/ATP carrier in complex with carboxyatractyloside. Nature. 426:39-44. 